Research article

\title{
Hypoxia-inducible factor-1 $\alpha$ and vascular endothelial growth factor expression in circulating tumor cells of breast cancer patients
}

\author{
Galatea Kallergi1, Harris Markomanolaki ${ }^{1}$, Vicky Giannoukaraki ${ }^{1}$, Maria A Papadaki ${ }^{1}$, Areti Strati ${ }^{2}$, \\ Evi S Lianidou², Vassilis Georgoulias ${ }^{1,3}$, Dimitris Mavroudis ${ }^{1,3}$ and Sofia Agelaki ${ }^{1,3}$
}

\author{
${ }^{1}$ Laboratory of Tumor Cell Biology, School of Medicine, University of Crete, Voutes, Heraklion, 71110, Greece \\ 2Laboratory of Analytical Chemistry, Department of Chemistry, University of Athens, 15771, Greece \\ ${ }^{3}$ Department of Medical Oncology, University General Hospital of Heraklion, Voutes, Heraklion, 71110, Greece \\ Corresponding author: Galatea Kallergi, kalergi@med.uoc.gr
}

Received: 4 May 2009 Revisions requested: 30 Jun 2009 Revisions received: 4 Nov 2009 Accepted: 17 Nov 2009 Published: 17 Nov 2009

Breast Cancer Research 2009, 11:R84 (doi:10.1186/bcr2452)

This article is online at: http://breast-cancer-research.com/content/11/6/R84

(c) 2009 Kallergi et al; licensee BioMed Central Ltd.

This is an Open Access article distributed under the terms of the Creative Commons Attribution License (http://creativecommons.org/licenses/by/ 2.0), which permits unrestricted use, distribution, and reproduction in any medium, provided the original work is properly cited.

\begin{abstract}
Introduction The detection of peripheral blood circulating tumor cells (CTCs) and bone marrow disseminated tumor cells (DTCs) in breast cancer patients is associated with a high incidence of disease relapse and disease-related death. Since hypoxiainducible factor-1 $\alpha$ (HIF-1 $\alpha$ ) and vascular endothelial growth factor (VEGF) play an important role in angiogenesis and tumor progression, the purpose of the current study was to investigate their expression in CTCs.

Methods The expression of cytokeratins (CK), VEGF, vascular endothelial growth factor receptor-2 (VEGF2), HIF-1 $\alpha$ and phosphorylated-focal adhesion kinase (pFAK) in CTCs from 34 patients with metastatic breast cancer who had detectable CK19 mRNA-positive CTCs was assessed using double staining experiments and confocal laser scanning microscopy. Peripheral blood mononuclear cells (PBMCs) were stained with a monoclonal A45-B/B3 pancytokeratin antibody in combination with either VEGF or VEGFR2 or HIF-1 $\alpha$ or pFAK antibodies, respectively.
\end{abstract}

Results pFAK expression in circulating tumor cells was detected in $92 \%$ of patients whereas expression of VEGF, VEGF2 and HIF-1 $\alpha$ was observed in $62 \%, 47 \%$ and $76 \%$ of patients, respectively. VEGF, VEGF2, HIF- $1 \alpha$ and pFAK were expressed in $73 \%, 71 \%, 56 \%$ and $81 \%$, respectively, of all the detected CTCs. Vascular endothelial growth mRNA was also detected by quantitative real-time RT-PCR in immunomagnetically-separated CTCs. Double and triple staining experiments in cytospins of immunomagneticallyisolated CTCs showed that VEGF co-expressed with HIF-1 $\alpha$ and VEGF2.

Conclusions The expression of pFAK, HIF- $1 \alpha$, VEGF and VEGF2 in CTCs of patients with metastatic breast cancer could explain the metastatic potential of these cells and may provide a therapeutic target for their elimination.

\section{Introduction}

The development of metastasis is mainly responsible for cancer-related death. Malignant cells detached from the primary tumor possessing advantageous biological characteristics are presumed to generate distant disease sites. Indeed, it has been shown that metastasis is associated with the presence of circulating (CTCs) and disseminated (DTCs) tumor cells in peripheral blood and bone marrow, respectively, of otherwise metastasis-free patients $[1,2]$. Several studies have reported that the detection of CTCs and DTCs represents a strong and independent prognostic factor for a decreased disease-free and overall survival [3-5]. In addition, recent studies have shown that in patients with metastatic breast cancer, the assessment of CTCs is an earlier and more reliable marker than that of DTCs, associated with disease prognosis and suitable for monitoring of tumor response to chemotherapy [6].

CTCs: circulating tumor cells; HIF-1 $\alpha$ : hypoxia inducible factor 1 alpha; HUVEC: human umbilical vein endothelial cells; PBMCs: peripheral blood mononuclear cells; PBS: Phosphate Buffered Saline solution; pFAK: phosphorylated-focal adhesion kinase; RT-PCR: reverse transcription-polymerase chain reaction; VEGF: vascular endothelial growth factor; VEGFR2: vascular endothelial growth factor receptor 2. 
Angiogenesis, primarily regulated by vascular endothelial growth factor (VEGF), is a critical event in tumor progression and metastasis [7]. Tumor cells produce and release vascular endothelial growth factor in response to oxygen and nutrients deprivation that in turn stimulates the formation of new vessels and promotes tumor growth and dissemination [8]. Besides its role in new blood formation, VEGF has been also shown to stimulate the proliferation of tumor cells. Indeed, VEGF enhanced the proliferation and the migration of breast cancer cells $[9,10]$, whereas siRNA targeting of VEGF in MCF7 breast cancer cell line, effectively inhibited cell proliferation and enhanced apoptosis [11]. Moreover, VEGF expression in breast cancer cells was correlated with decreased response to hormone treatment [9] and with reduced survival in breast cancer patients [12]. Focal adhesion kinase (FAK) and Src catalytic activities are important in promoting VEGF-induced tumor angiogenesis [13] whereas the inhibition of FAK reduces VEGF expression resulting in small avascular tumors in mice [14].

VEGFR2 is the main receptor for VEGFs and its action is related to the activation of signalling molecules such as PLC $\gamma 1$, phosphoinositide-3 kinase (PI-3 kinase), Akt, Src and ERK [15].

The expression of VEGF in cancer cells is under the control of hypoxia-inducible factor- $1 \alpha$ (HIF- $1 \alpha$ ) which is a transcription factor induced under hypoxia conditions [16]. In the absence of oxygen, HIF-1 $\alpha$ binds to hypoxia-response elements inducing the transcription of VEGF gene among other factors such as LDH-A, NOS, EPO and so on [16]. The expression of HIF$1 \alpha$ depends on FAK and phosphoinositide-3 kinase (PI-3) activation in cancer cells [17].

Our group has recently demonstrated that FAK as well as PI3 and Akt kinases are phosphorylated and thus activated in CTCs of breast cancer patients $[18,19]$. Since FAK is implicated in the angiogenesis process and induces the expression of VEGF, it was of interest to evaluate whether CTCs from breast cancer patients have activated the angiogenesis pathway by expressing HIF- $1 \alpha$ and VEGF. This could be an important mechanism associated with the metastatic potential of these cells and therefore could bear important therapeutic implications.

\section{Materials and methods Cell cultures}

All cell lines were obtained from ATCC (American Type Culture Collection, USA). The MCF7 mammary adenocarcinoma cells were cultured in 1:1 (v/v) Dulbecco's Modified Eagle (DMEM)/Ham's F12 medium (GIBCO-BRL NY, USA), supplemented with 10\% fetal bovine serum (FBS), $2 \mathrm{mM} \mathrm{L-}$ glutamine, $30 \mathrm{mM} \mathrm{NaHCOB}{ }_{3 B}, 16 \mathrm{ng} / \mathrm{ml}$ insulin and $50 \mathrm{mg} / \mathrm{ml}$ penicillin/streptomycin. MDA-MB-231 and human umbilical vein endothelial cells (HUVEC) cells were cultured in DMEM
10\% FBS whereas the MDA-MB-453, SKBR3 and T47D cells were cultured in RPMI 10\% FBS. Subcultivation of all cell lines was performed with $0.25 \%$ trypsin and $5 \mathrm{mM}$ EDTA. These cell lines were used as positive controls for VEGF, vascular endothelial growth factor receptor 2, HIF-1 $\alpha$ and phosphorylated-focal adhesion kinase ( $\mathrm{pFAK}$ ) expression. Cells were maintained in a humidified atmosphere of $5 \% \mathrm{COB}_{2 \mathrm{~B}}$ in air. All experiments were performed during the logarithmic growth phase of the cells. Fifteen to $20 \mathrm{~h}$ prior to the experiments, cells were transferred to serum-free culture medium containing only L-glutamine, $\mathrm{NaHCOB}_{3 \mathrm{~B}}$ and penicillin/streptomycin. HUVEC cells were used as a positive control for HIF-1 $\alpha$ expression [20].

\section{Patient samples and cytospin preparation}

Blood samples obtained before the initiation of the respective line of treatment from 34 patients with metastatic breast cancer and tumor cells circulating in their peripheral blood as detected by real-time PCR for Cytokeratin (CK) -19 mRNA [21], were analyzed. Peripheral blood (10 $\mathrm{ml}$ in EDTA) was drawn from the middle of vein puncture after the first $5 \mathrm{ml}$ of blood were discarded. This precaution was undertaken in order to avoid contamination of the sample with epithelial cells from the skin during sample collection. All patients gave their informed consent to participate in the study, which has been approved by the Ethics and Scientific Committees of our institution.

Peripheral blood mononuclear cells (PBMC) were isolated with Ficoll-Hypaque density gradient $(d=1,077 \mathrm{~g} / \mathrm{mol})$ centrifugation at $1,800 \mathrm{rpm}$ for 30 minutes. PBMCs were washed three times with Phosphate Buffered Saline solution (PBS) and centrifuged at $1,500 \mathrm{rpm}$ for 10 minutes. Aliquots of 250,000 cells were centrifuged at $2,000 \mathrm{rpm}$ for two minutes on glass slides. Cytospins were dried up and stored at $-80^{\circ} \mathrm{C}$. Four to five slides were evaluated from the same blood sample were evaluated for each examined molecule in the corresponding double staining experiments. The results were expressed as the mean number of CTCs/250,000 PBMCs.

\section{Confocal laser scanning microscopy}

The presence of CK-positive cells in PBMCs of cytospin preparations was investigated using the A45-B/B3 (Micromet, Munich, Germany) mouse antibody (detecting CK8, CK18 and CK19). Cytospins were also double stained with antiCD45 (common leukocyte antigen) antibody to exclude possible illegitimate expression of cytokeratins on hematopoietic cells. The cytomorphological criteria (high nuclear to cytoplasmic ratio, larger than white blood cells and so on) proposed by Meng et al [22] were used in order to characterize a CK-positive cell as a CTC. Cytospins from the same patients were also stained with goat anti-VEGF (R\&D Systems, Inc, Minneapolis, USA), rabbit anti-VEGFR2 (Cell Signaling, Boston, USA), rabbit anti-HIF-1 $\alpha$ (Santa Cruz, Santa Cruz, CA, USA) and mouse anti-pFAK (Chemicon, Temecula, CA, USA) anti- 
bodies in double staining experiments and the expression of these molecules was assessed using confocal laser scanning microscopy [18] (see additional data file 1). Double staining experiments validating VEGF, VEGFR2 and HIF-1 $\alpha$ expression in 10 healthy blood donors were also performed as controls. Control experiments for pFAK expression have been previously described [18].

For double staining experiments PBMC cytospins were fixed with $3 \%$ paraformaldehyde (PFA) for 30 minutes. Cell membrane permeabilization was performed with $0.5 \%$ Triton for 10 minutes followed by overnight incubation with blocking buffer (PBS/1\%BSA). Subsequently, slides were stained for cytokeratin with the A45-B/B3 antibody and for VEGF, VEGFR2 or HIF-1 $\alpha$ with the corresponding antibodies for 45 minutes. Cells were then incubated with the appropriate secondary antibody for one hour. Finally, slides were stained with DAPI conjugated with antifade (Invitrogen, Carlsbad, CA, USA). Phospho-FAK ( $p F A K$ ) staining was performed as previously described [18]. Samples were analyzed using a confocal laser scanning microscope module (Leica Lasertechnik, Heidelberg, Germany) and images were analyzed with the respective software.

Triple immunofluoresence for CK/VEGF/HIF-1 $\alpha$ and CK/ VEGF/VEGFR2 was also performed. Cells were initially fixed using $4 \%$ formaldehyde for 15 minutes at room temperature (RT). Permeabilization was achieved with $0.1 \%$ Triton X-100 for five minutes at RT. After blocking with PBS supplemented with 10\% (v/v) FBS for 30 minutes, cells were incubated with the corresponding antibodies for 45 minutes each. Zenon technology (Fluorescein Isothiocyanate (FITC)-conjugated IGg1 antibody) (Molecular Probes, Invitrogen, Carlsbad, CA, USA) was used for CK detection with the A45-B/B3 antibody. Zenon antibodies were prepared within $30 \mathrm{~min}$ before use. Zenon reagent was incubated with A45-B/B3 for five minutes, blocking buffer was added for $5 \mathrm{~min}$ and the conjugated antibodies were then ready for use.

VEGFR2 was detected using anti-rabbit antibody labelled with Alexa555 (Molecular Probes, Invitrogen, Carlsbad, CA, USA), whereas VEGF was detected by anti-mouse antibody (Santa Cruz, Santa Cruz, CA, USA) labelled with Alexa 633 (Molecular Probes, Invitrogen, Carlsbad, CA, USA). For CK/VEGF/ HIF-1 $\alpha$ staining, zenon technology (FITC-conjugated) was also used for CK detection. For VEGF, an anti-mouse antibody labelled with Alexa 633 was used and HIF-1 $\alpha$ was stained with anti-rabbit antibody labelled with Alexa555. Cells incubated with the different antibodies were post-fixed with 4\% (v/ v) formadehyde in PBS for 15 minutes at RT. Finally, cells were stained with DAPI conjugated with antifade.

\section{Immunomagnetic separation of CTCs}

In 10 patients with metastatic breast disease, a negative selection procedure was performed for the isolation of CTCs according to Naume et al [23]. Briefly, $100 \mu$ of CELLection beads coated with anti-CD45 monoclonal antibody (Dynal, Invitrogen, Carlsbad, CA, USA) were added in $10^{7 \mathrm{P}} / \mathrm{ml}$ PBMCs in PBS/0.1\% BSA/2 mM EDTA. PBMCs were isolated from $20 \mathrm{ml}$ of blood using Ficoll density gradient centrifugation. After an incubation for 30 minutes at $4^{\circ} \mathrm{C}$, the supernatant was transferred in FBS-coated tubes and cells were cyto-centrifuged at $2000 \mathrm{rpm}$ for two minutes on glass slides. The same number of cells was centrifuged at 1,500 rpm for five minutes and the pellet was stored at $-80^{\circ} \mathrm{C}$ for RNA extraction.

In control spiking experiments, 10 MCF7 tumor cells $\left(1 / 10^{6}\right)$, 100 cells $\left(10 / 10^{6}\right)$ and 1,000 cells $\left(100 / 10^{6}\right)$ were spiked in $10^{7}$ PBMCs obtained from healthy volunteers. Subsequently, $100 \mu \mathrm{l}$ of anti-CD45-beads were added in every $10^{7}$ PBMCs according to the manufacturer's instructions and cytospins were prepared using the isolated cells. By this method, it was possible to estimate the enrichment of CTCs per 250,000 PBMCs. The proportion of CTC/PBMCs after enrichment was approximately $1 / 60$ and the obtained degree of CTCs enrichment ranged from $150 \%$ to $300 \%$. Moreover, in order to verify that the tumor cell-depleted PBMCs fraction did not contain CTCs, immunostaining with A45-B/B3 antibody was performed on cytospins prepared from the CTCs-depleted fraction. Three independent spiking experiments demonstrated that no CK-positive cells could be identified in these samples.

\section{Immunoblot analysis}

MCF7, T47D, SKBR3, MDA-MB-453 and MDA-MB-231 cells were lysed in $100 \mu$ lysis buffer each $(50 \mathrm{mM}$ Tris/ $\mathrm{HCl}, 1 \%$ TritonX-100 pH 7.4, 1\% Sodium deoxycholate, 0.1\% SDS, $0.15 \% \mathrm{NaCl}, 1 \mathrm{mM}$ EDTA, $1 \mathrm{mM}$ sodium orthovanadate) at $4^{\circ} \mathrm{C}$ and flasks were scrapped off. The remaining insoluble material was removed by centrifugation. Protein concentration of the samples was determined using the Bio-Rad protein kit. Equal amounts of protein $(100 \mu \mathrm{g})$ were separated with SDS electrophoresis and blotted onto nitrocellulose membrane. Proteins were incubated with anti-VEGF, anti-VEGFR2 and HIF- $1 \alpha$ antibodies for $1 \mathrm{~h}$ at room temperature and then with the appropriate secondary antibody. Detection of protein bands was succeeded using the ECL kit. Western blots were quantified using Bio-Rad Quantity One program. The numbers we provided correspond to the Adj Vol Intensity per $\mathrm{mm}^{2}$ and the measurements concerned equal square area. Intensity of the background was set as 0 .

\section{Real-time RT-PCR for VEGF quantification}

RNA was extracted, from both the immunomagnetically CTCenriched and CTC-depleted PBMCs fractions, using the Trizol reagent (Invitrogen, Carlsbad, CA, USA). The same procedure was followed for three normal volunteers. Both fractions were used in order to investigate the relative expression of VEGF since it could not be excluded that some hemopoietic cells had contaminated the CTC-enriched fraction. All preparations 
and handling of RNA took place in a laminar flow hood under RNase-free conditions. RNA concentration was quantified with Nanodrop ND-1000 spectophotometer. Afterwards equal RNA amounts from patients' CTCs-enriched and CTCsdepleted PBMCs proceeded to CDNA synthesis with Superscript III platinum kit (Invitrogen, Carlsbad, CA, USA) according to manufacturer's instructions. Real-time RT-PCR for VEGF quantification was performed as previously described [24].

\section{Results \\ Patient characteristics}

Thirty-four patients with metastatic breast cancer and CK-19 mRNA positive CTCs detected in their peripheral blood [21], were enrolled in the present study. Patient characteristics are listed in Table 1. Median age was 57 (range, 30 to 76) years and $88 \%$ of the patients were post-menopausal. Twenty-three $(68 \%)$ patients had relapsed after treatment for early breast cancer. Adjuvant chemotherapy regimens included anthracyclines in four patients, taxanes and anthracyclines in 10, Cyclophosphamide, Methotrexate and 5-Fluoruracil (CMF regimen) in six patients, whereas in three the type of adjuvant chemotherapy was unknown. Eighteen (53\%) patients had received no prior chemotherapy for metastatic disease, seven (21\%) had received one and nine (26\%) two or more prior lines of treatment. Patients had not received antiangiogenic agents as part of their treatment before study entry. Patients with HER2 positive tumors received chemotherapy in combination with trastuzumab or lapatinib. Second- and third- (or higher) line regimens administered included anthracyclines or taxanes as single agents or combinations of docetaxel and capecitabine, taxanes and antracyclines, vinorelbine and gemcitabine and vinorelbine in combination with trastuzumab or lapatinib in patients with HER2 positive tumors.

VEGF and VEGFR2 expression in breast cancer cell lines In preliminary experiments, we investigated the expression of VEGF, VEGFR2 HIF-1 $\alpha$ and pFAK in breast cancer cell lines. Among the five examined breast cancer cell lines MDA-MB231, MDA-MB-453 and SKBR3 cells are generally considered more aggressive compared to T47D and MCF7 cells [2527]. Immunoblot analysis of equal protein amounts of cell lysates with anti-VEGF antibody revealed that the less-aggressive cell lines (T47D and MCF7) expressed less VEGF than the more aggressive cell lines SKBR3, MDA-MB-231 and MDA-MB-453 cells (Figure $1 \mathrm{~A}$ ). HIF- $1 \alpha$ and pFAK was expressed in all the examined cell lines except MDA-MB-231, whereas VEGFR2 was mainly expressed in MDA-MB-231 (see additional data file 2).

Subsequently, the sensitivity and specificity of immunofluorescence assays for the detection of VEGF, VEGFR2 and HIF-1 $\alpha$ in CTCs were evaluated on cytospins of tumor cells spiked in PBMCs obtained from healthy volunteers.

\section{VEGF and VEGFR2 expression in CTCs}

Using double staining experiments, VEGF-expressing CK-positive CTCs were detected in $21(62 \%)$ patients (Figure 1BI, II, III, see also additional data file 3 ). Among a total of 99 CK-19 positive CTCs recognized in cytospins from all patients, 72 (73\%) stained positively for both VEGF and CK (Figure 1C) showing that the majority of the detected CTCs expressed VEGF in their cytoplasm. Moreover, in 12 (35\%) patients all the detected CTCs expressed VEGF in their cytoplasm. The median percentage of CTCs expressing VEGF among the 34 patients was $100 \%$ (range; $29 \%$ to $100 \%$ ).

Consequently, VEGFR2-expressing $\mathrm{CK}^{+} \mathrm{CTC}$ s were identified in $16(47 \%)$ out of 34 patients (Figure 2Alll, see also additional data file 3). Among a total of $124 \mathrm{CK}^{+} \mathrm{CTCs}$ detected in all studied patients, $89(71 \%)$ were double stained [(CK $(+) /$ VEGFR2(+)] (Figure 1C). In only four (12\%) patients, all the detectable CTCs expressed VEGFR2. The median expression of VEGFR2 on CTCs identified per patient was 75\% (range, $10 \%$ to $100 \%)$. Confocal laser scanning microscopy revealed that VEGFR2 was mainly localized adjacent to the plasma membrane (Figure 2AI); however, in some CTCs, VEGFR2 was also intracellulary distributed (Figures $2 \mathrm{All}, 3 \mathrm{BI}$ and $3 \mathrm{Cl}$ ). Spearman's analysis showed a significant correlation $(P=$ 0.042 ) between the number of VEGF- and VEGFR2-expressing CTCs in the whole group of patients. No double positive cells $\left(\mathrm{CK}^{+} \mathrm{VEGF}^{+}\right.$or $\left.\mathrm{CK}^{+} \mathrm{VEGFR2}{ }^{+}\right)$could be observed in healthy volunteers ( $n=10$ normal blood donors), although VEGF and VEGFR2 expression was detected in some hematopoietic cells.

The expression of either VEGF or VEGFR2 on CTCs did not correlate with ER/PR or HER2 status of the primary tumor. In addition, no correlation was found between VEGF and VEGFR2 expression and line of treatment (no prior regimens vs one or more regimens for metastatic disease) or response to treatment.

\section{Expression of HIF-1 $\alpha$ and phosphorylated FAK in CTCs} VEGF expression is under the regulation of HIF- $1 \alpha$ transcription factor and of FAK [28]. In order to determine whether angiogenic pathways including HIF- $1 \alpha$ and FAK operate on CTCs, the expression of these molecules was assessed using double staining experiments in patient's cytospins.

Figure 2BIII demonstrates that $26(76 \%)$ out of 34 patients had HIF- $1 \alpha^{+} / \mathrm{CK}^{+}$-positive cells. HIF- $1 \alpha$ is distributed both in the cytoplasm and in the nucleus of the majority of CTCs (Figure $2 \mathrm{BI}$ and $2 \mathrm{BII}$, see also additional data file 3 ). In nine (26\%) patients, all the detected CTCs expressed HIF-1 $\alpha$; the median proportion of cells expressing HIF- $1 \alpha$ was $69 \%$ (range; $5 \%$ to $100 \%)$. Among a total of 223 CK-positive cells detected in all studied patients, $125(56 \%)$ were double-positive (CK+/HIF$\left.1 \alpha^{+}\right)$(Figure 1C). HIF-1 $\alpha$-positive CTCs co-exist with VEGF- 
Table 1

Patient characteristics

\section{Age}

Median (range)

Menopausal status

Premenopausal

Postmenopausal

Primary breast cancer at presentation

Tumor size (cm)

$1-1.9 / 2-4.9 / \geq 5 / \mathrm{UN}$

Lymph nodes

$0 / 1-3 / \geq 4 / U N$

Grade

II/III/UN

Disease sites ${ }^{*}$

2

3

$\geq 4$

Unknown

Predominantly visceral disease

Yes

No

Unknown

Line of treatment **

0

1

$\geq 2$

ER/PR status

Positive

Negative

Unknown

HER2 status

Positive $^{\star \star \star}$

Negative

Unknown

$\mathrm{UN}=$ unknown

* Numbers correspond to the number of organs involved

** Numbers correspond to the number of prior therapies administered for metastatic disease

*** $\mathrm{IHC:3+}$ or FISH positive
57 (30 to 76$)$

n (\%)

4 (12)

30 (88)

$23(68)$

$3(13) / 14(61) / 2(9) / 4(17)$

$7(30) / 6(26) / 8(35) / 2(9)$

$8(35) / 11(48) / 4(17)$

$5(15)$

$15(44)$

5 (15)

8 (23)

1 (3)

11 (32)

$22(64)$

1 (3)

$18(53)$

7 (21)

$9(26)$

$16(47)$

$13(38)$

5 (15)

7 (20)

$23(68)$

4 (12) 
Figure 1

(a)

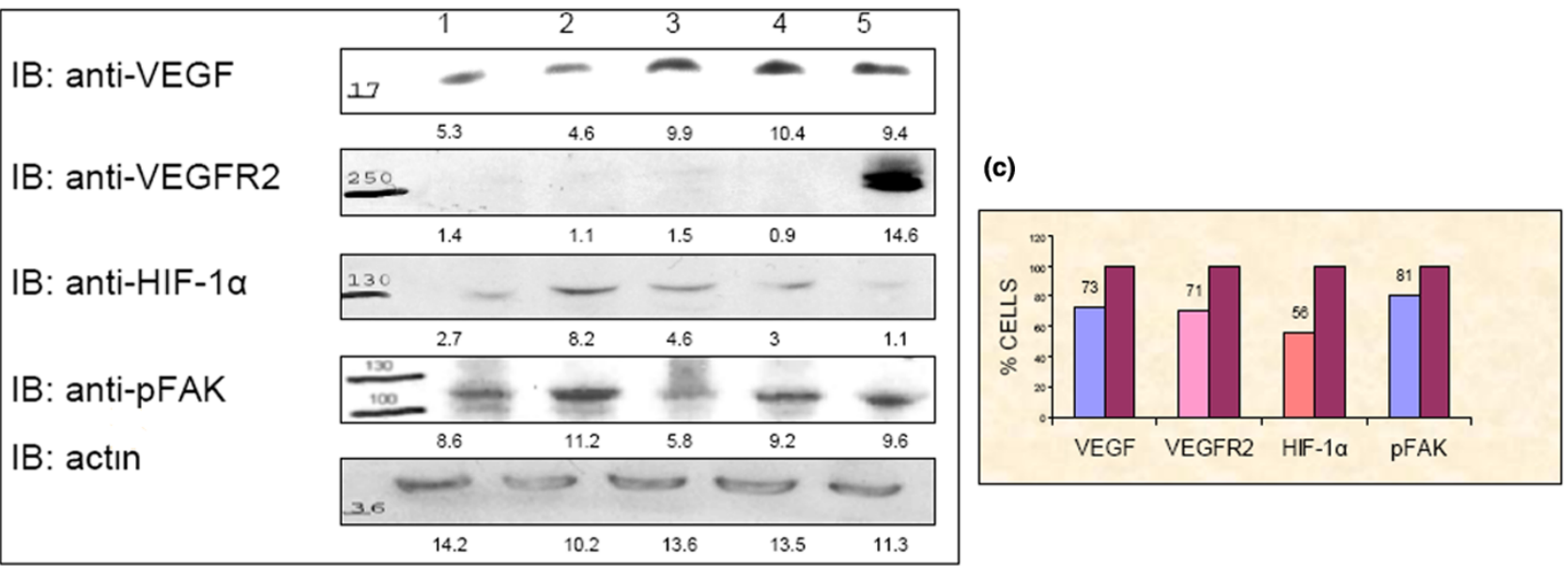

(b)

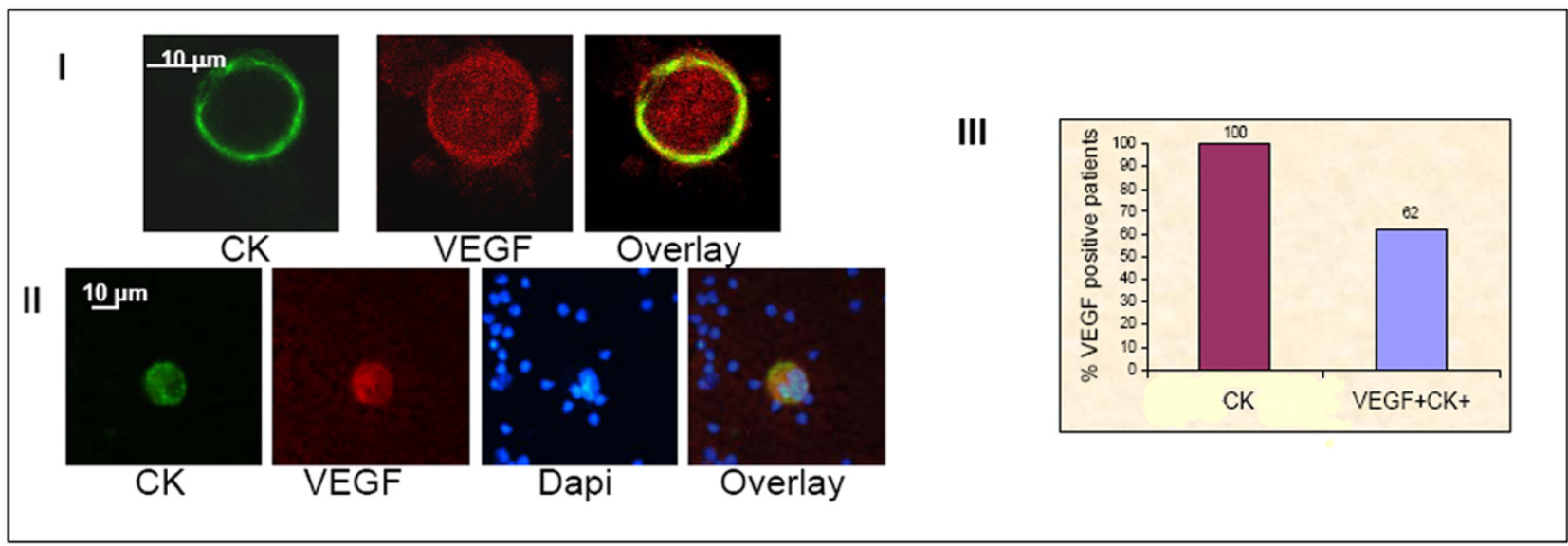

Vascular endothelial growth factor expression in breast cancer cell lines and in circulating tumor cells. A: VEGF, VEGFR2 HIF-1 $\alpha$ and pFAK expression in cultured breast cancer cell lines, 1: MCF7, 2:T47D, 3: SKBR3 4:MDA MB-453, 5: MDA MB-231 (western blot analysis of equal protein amount of cell lysates). B (I): Representative images of confocal laser scanning microscopy of PBMCs cytospins stained with pancytokeratins A45 B/B3 (green), and VEGF anti-goat (red) antibodies. B (II): Representative images of PBMCs cytospins stained with pancytokeratins A45 B/B3 (green), VEGF anti-goat (red) antibodies and Dapi (blue). B (III): Quantification of VEGF and CK co-expression in CTCs of 34 metastatic breast cancer patients. All the examined patients (100\%) presented CTCs in their blood, while $62 \%$ also presented double positive cells (VEGF+CK+). (C) Quantification of double positive CTCs/total CTCs for each examined molecule in 34 metastatic breast cancer patients. For pFAK testing cytospins from 12 patients were evaluated. indicatively. Seventy-three percent of the examined CTCs were VEGF+CK+ positive, $71 \%$ were VEGFR2 ${ }^{+}{ }^{+}{ }^{+}$, $56 \%$ were $\mathrm{HIF}-1 \alpha^{+} \mathrm{CK}^{+}$and $81 \%$ were $\mathrm{pFAK}^{+} \mathrm{CK}^{+}$.

or VEGFR2-positive CTCs in 16 (47\%) and 14 (41\%) breast cancer patients, respectively.

Double staining experiments in the control group of 10 healthy blood donors $(n=10)$, revealed no CK-positive cells expressing HIF- $1 \alpha$ in their PBMCs, whereas this transcription factor was expressed in some hematopoietic cells.

We have recently reported that pFAK was expressed in all CKpositive CTCs of patients with early breast cancer [18]. In the present study we investigated the expression of pFAK in 12 patients with metastatic disease for whom adequate material was available. Double staining experiments revealed that pFAK was expressed in 92\% (11 out of 12) of the CTCs-positive patients (Figure $3 \mathrm{Al}$ and $3 \mathrm{All}$ ). Among a total of $26 \mathrm{CK}^{+}$ CTCs, 21 (81\%) were double stained $\left(\mathrm{CK}^{+} \mathrm{pFAK}{ }^{+}\right.$) (Figure $1 \mathrm{C})$. In eight (67\%) out of 12 patients, all their CTCs were pFAK-positive. All of these patients also had HIF- $1 \alpha$-positive CTCs (Spearman's rho correlation analysis $P<0.001$ ). The median percentage of cells expressing pFAK was $100 \%$ (range 50\% to 100\%). No correlation was also found between the expression of HIF-1 $\alpha$ on CTCs and ER/PR or HER2 status of the tumor as well as with the line of treatment or response to treatment. 
Figure 2

(a)

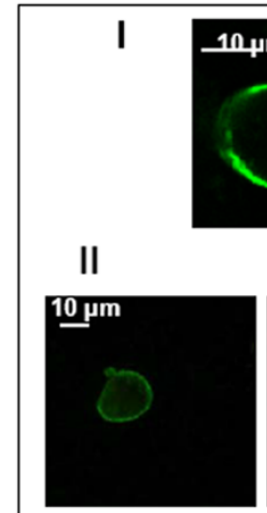

CK

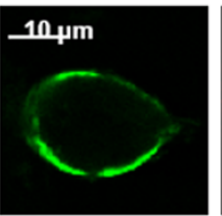

CK

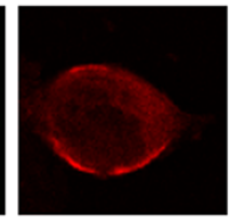

VEGFR2

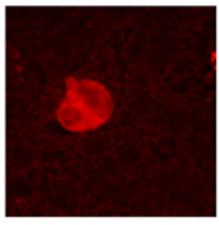

VEGFR2

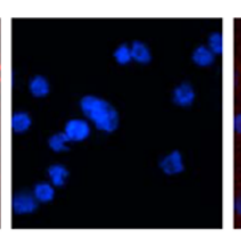

Dapi

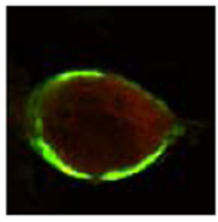

Overlay

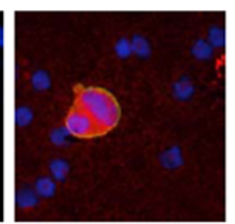

Overlay
III

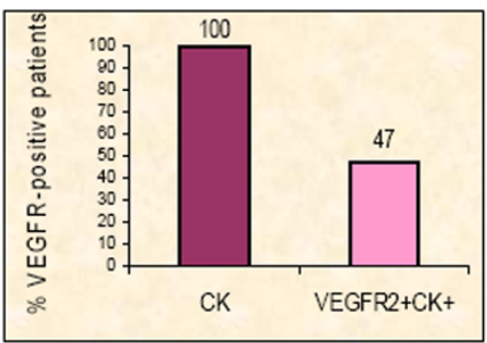

(b)
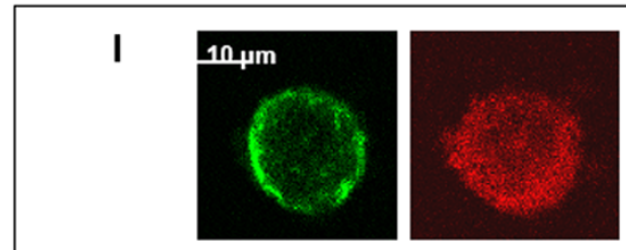

CK

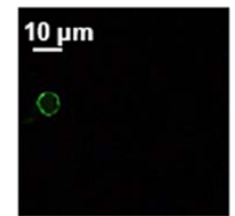

CK

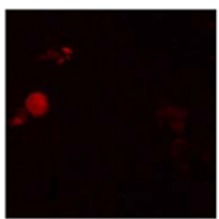

HIF-1a
HIF-1a

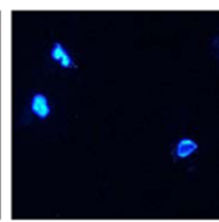

Dapi

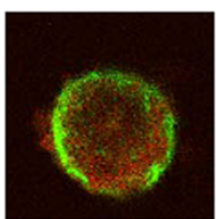

Overlay

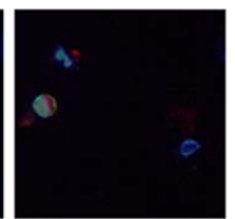

Overlay
III

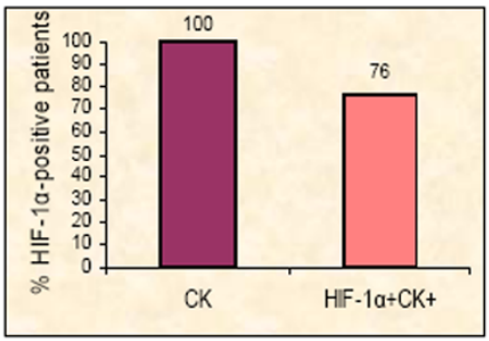

Vascular endothelial growth factor receptor 2 and hypoxia-inducible factor-1 $\alpha$ in circulating tumor cells of patients with breast cancer. A (I): Representative images of cytospin double stained with monoclonal pancytokeratin (A45-B/B3) (green) and polyclonal VEGFR2 anti-rabbit (red) antibodies. A (II): Representative images of PBMCs cytospins stained with pancytokeratins A45 B/B3 (green), VEGFR2 anti-rabbit (red) antibodies and Dapi (blue). A (III): Quantification of VEGFR2 and CK co-expression in CTCs of 34 patients with metastatic breast cancer. CTCs were detected in all (100\%) patients while in $47 \%$ double positive cells (VEGFR2 $+\mathrm{CK}^{+}$) were identified. B (I): Representative image of a cytospin double stained with monoclonal pancytokeratin (A45-B/B3) (green) and polyclonal HIF-1 $\alpha$ (red) antibodies. B (II): Representative images of PBMCs cytospins stained with pancytokeratins A45 B/B3 (green), HIF-1 $\alpha$ anti-rabbit (red) antibodies and Dapi (blue). B (III): Quantification of HIF-1 $\alpha$ and CK co-expression in CTCs of 34 patients with metastatic breast cancer. All the examined patients $(100 \%)$ harvested CTCs in their blood, while $76 \%$ also presented double positive cells (HIF- $1 \alpha^{+} \mathrm{CK}^{+}$).

Negative and positive controls were used in each experiment for every examined molecule Figure $4 \mathrm{~A}, \mathrm{~B}$ and $4 \mathrm{C}$.

\section{Co-expression of VEGF, VEGFR2 and HIF- $1 \alpha$ in CTCs}

The co-expression of the examined molecules was further analysed in 10 patients after negative immmunomagnetic separation. Negative selection was preferred because prior experiments using MCF7 cells spiked into PBMCs obtained from healthy donors to estimate recovery yields, revealed higher recovery rates for negative compared to positive selection, even in low cell concentrations (one CTC in $10^{6}$ PBMCs); moreover, the quality of RNA (ratio 260:280) extracted from
CTCs-enriched and CTCs-depleted cell fractions was superior after negative compared to positive immunomagnetic selection (data not shown).

The expression of CK/VEGF, CK/VEGFR2 and CK/HIF-1 $\alpha$ was investigated in PBMCs cytospins before and after immmunomagnetic separation. Figure $3 \mathrm{BI}$, II shows that VEGF could be co-localized with VEGFR2 or HIF-1 $\alpha$ in the same cell.

In order to further confirm that HIF-1 $\alpha$, VEGF and VEGFR2 were co-expressed in the same CTC and not in residual PBMCs, triple immunofluorence CK/VEGF/VEGFR2 and CK/ 
Figure 3

(a)

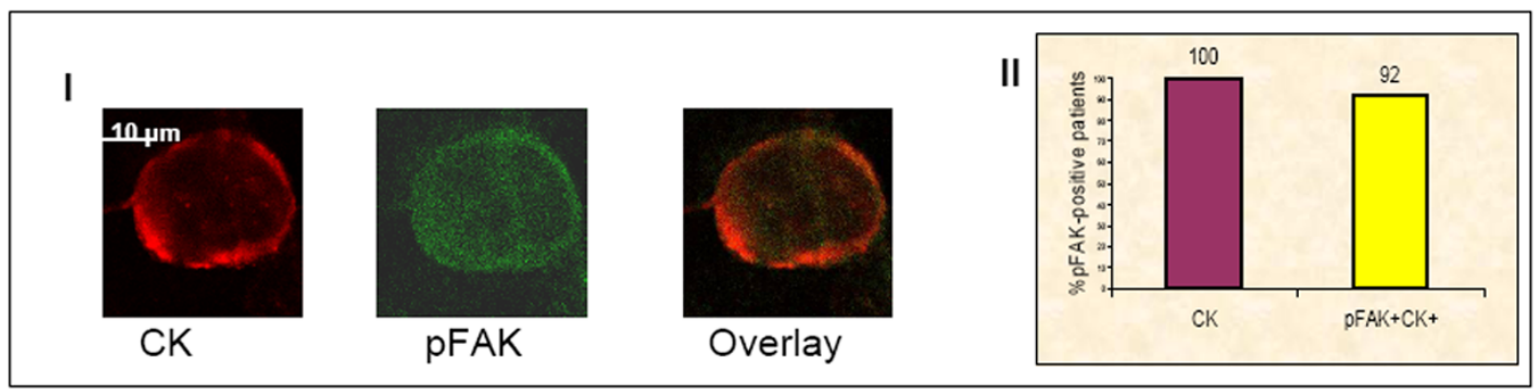

(b)

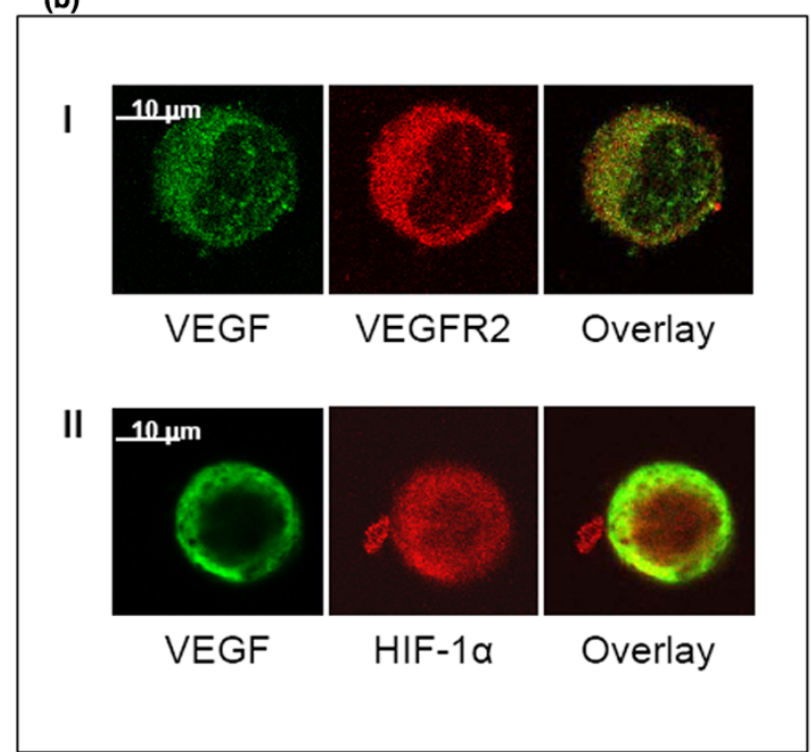

(c)

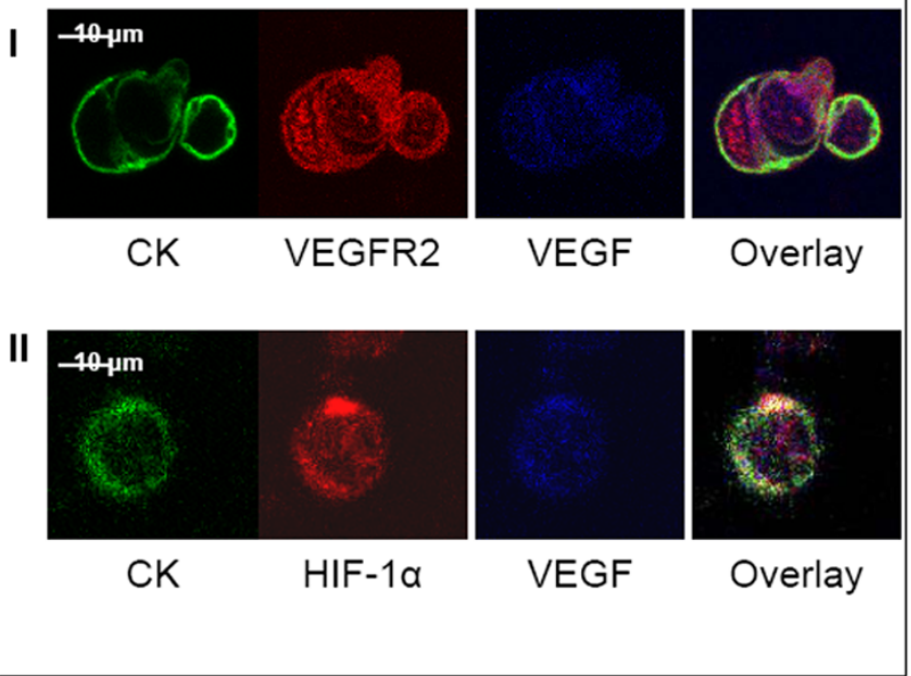

Phosphorylated-focal adhesion kinase expression in circulating tumor cells and co-expression of vascular endothelial growth factor/vascular endothelial growth factor receptor or vascular endothelial growth factor/hypoxia-inducible factor-1 $\alpha$ in circulating tumor cells of patients with breast cancer. A (I): Representative image of a cytospin double stained with polyclonal pancytokeratin (red) and (monoclonal) pFAK (green) antibodies. A (II): Quantification of pFAK and CK co-expression in CTCs of 12 patients with metastatic disease. All the examined patients (100\%) presented CTCs in their blood, while $92 \%$ also presented double positive cells ( $\left.\mathrm{pFAK}+\mathrm{CK}^{+}\right)$. B: Representative confocal laser scanning micrographs of $\mathrm{CTCs}$ cytospins after negative immunomagnetic separation in 10 patients with metastatic breast cancer. Cells cytospins were double stained with VEGF and either (I) VEGFR2 or (II) HIF-1 $\alpha$ antibodies, respectively. C: Representative image of a cytospin, triple stained with monoclonal pancytokeratin (A45-B/B3) (green) and VEGF antibodies (blue) along with either (I) VEGFR2 or (II) HIF-1 $\alpha$ (B) (red) polyclonal antibodies (red).

VEGF/HIF-1 $\alpha$ was performed in the same patients. Figure $3 \mathrm{Cl}$, II demonstrates that VEGF is co-expressed with VEGFR2 and HIF-1 $\alpha$ in CTCs.

\section{VEGF mRNA expression in CTCs}

To determine whether VEGF was produced by the CTC and not adsorbed from the plasma, RNA extracted from both the CTCs-enriched and the corresponding CTCs-depleted cell fractions were analyzed for VEGF using equal RNA amount of each fraction and quantitative real-time RT-PCR assay as pre-

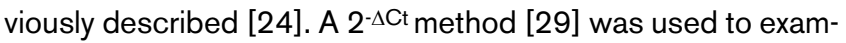
ine the relative expression of VEGF in CTCs-enriched fraction compared to the CTCs-depleted fraction.

The results revealed that VEGF mRNA was overexpressed in four $(40 \%)$ out of 10 examined patients (compared to corre- sponding fractions of normal blood donors) with a 3.1-, 8.3-, 11.5- and 25.3-fold increase in the CTCs-enriched cell fraction compared to the CTCs-depleted cell fraction. Double staining experiments with VEGF and A45/B-B3 antibodies confirmed the presence of intracytoplasmic VEGF in these four patients as well as in three additional patients (Table 2).

\section{Discussion}

Circulating and disseminated tumor cells derived from the primary tumor and possessing advantageous biological characteristics could acquire the capability of generating metastasis. CTCs have significant prognostic value for early and metastatic breast cancer patients $[4,5,30]$. These cells are very heterogenous and not fully characterized as yet [31,32]. VEGF is an important angiogenic factor for tumor vascularization. The expression of VEGF is under HIF- $1 \alpha$ control, in hypoxia condi- 
(a)

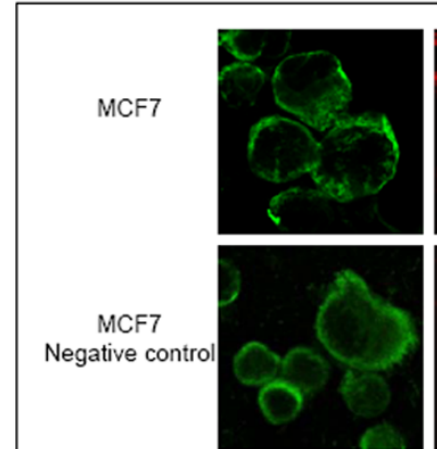

CK
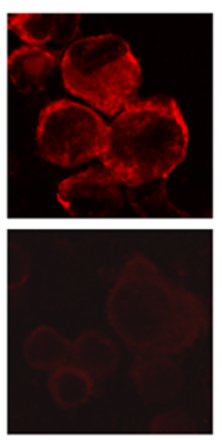

VEGF
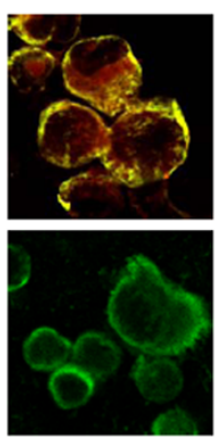

Overlay (b)

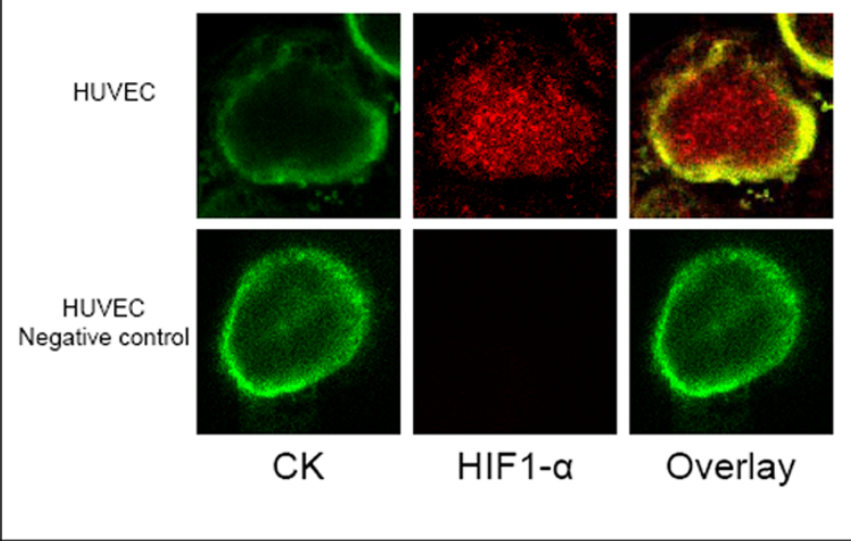

(c)

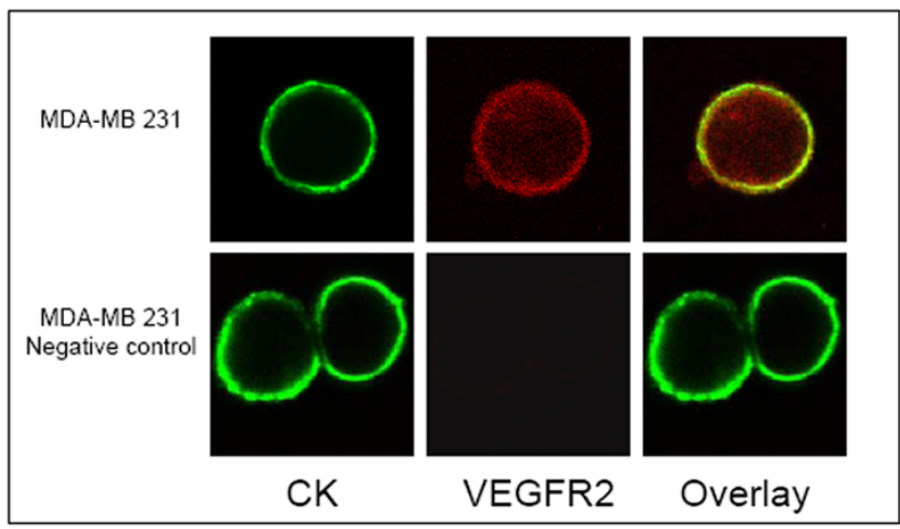

Vascular endothelial growth factor, vascular endothelial growth factor receptor 2 and hypoxia-inducible factor-1 $\alpha$ expression in MCF7, Human umbilical vein endothelial cells and MDA-MB-231 respectively. A: MCF7 cells stained with pancytokeratin/FITC (green) and VEGF/Alexa555 (red) or MCF7 cells stained with pancytokeratin/FITC (green) and just Alexa555 antibody without VEGF antibody (negative control). B: HUVEC cells stained with pancytokeratin/FITC (green) and HIF1- $\alpha /$ Alexa555 (red) or HUVEC cells stained with pancytokeratin/FITC (green) and just Alexa555 antibody without HIF1- $\alpha$ antibody (negative control). C: MDA-MB-231 cells stained with pancytokeratin/FITC (green) and VEGFR2/Alexa555 (red) or MDAMB-231 cells stained with pancytokeratin/FITC (green) and just Alexa555 antibody without VEGFR2 antibody (negative control).

tions [8,11]. In addition, focal adhesion kinase is also an upstream regulator of VEGF and HIF-1 $\alpha$ expression $[13,17]$. We have recently reported that phosphorylated-FAK was expressed in CTCs of all CK-positive early breast cancer patients [18]. In the present study we examined the existence of angiogenic molecules in CTCs of metastatic breast cancer patients.

Initially, we evaluated the expression of VEGF in breast cancer cell lines. As shown in Figure 1, VEGF was expressed in the cytoplasm of all the examined breast cancer cells. Interestingly, a correlation was evident between VEGF expression and cell aggressiveness. Specifically, the SKBR3, MDA-MB-231 and MDA-MB-453 cell lines which are highly metastatic [2527] expressed higher levels of VEGF compared to MCF7 and T47D which are estrogen receptor-positive and less aggres- sive cells. On the contrary, the total protein amount of HIF-1 $\alpha$ didn't follow the same pattern of expression. This is probably attributed to the fact that cells were cultured under normal oxygen conditions. Moreover, HIF- $1 \alpha$ is a transcription factor and it is possible that its activity relates to its nuclear distribution rather than the total intracellular amount of protein.

Since VEGF is related to the metastatic potential of cancer cells, we investigated VEGF expression in CTCs of 34 metastatic CK-positive breast cancer patients. The results of the current study revealed that VEGF was expressed in CTCs of $62 \%$ of the patients and the great majority (73\%) of these cells were double positive $\left(\mathrm{CK}^{+} \mathrm{VEGF}+\right.$, implying the possible involvement of VEGF in the metastatic potential of these cells. Indeed, in the study by Mohammed et al [25-27], VEGF expression on breast cancer cells was associated with 
Table 2

VEGF mRNA expression in CTCs of patients with metastatic breast cancer

\begin{tabular}{cccc}
\hline No Patients & $\mathbf{C t}_{(\mathrm{CTCs})}$ & $\mathbf{C t}_{(\mathrm{PBMCs})}$ & $\mathbf{2}^{-\Delta \mathrm{Ct}}$ \\
\hline 1 & 32,6 & 30,6 & 0,3 \\
2 & 33,7 & 36,8 & $\mathbf{8 , 3}$ \\
3 & 24,1 & 28,7 & $\mathbf{2 5 , 3}$ \\
4 & 37,9 & 38,8 & 1,8 \\
5 & 41,0 & 41,0 & 1,0 \\
6 & 39,4 & 41,0 & $\mathbf{3 , 1}$ \\
7 & 34,8 & 32,3 & 0,2 \\
8 & 41,0 & 36,9 & 0,1 \\
9 & 35,7 & 32,2 & 0,1 \\
10 & 34,7 & 38,2 & $\mathbf{1 1 , 5}$ \\
\hline
\end{tabular}

A 2- $\Delta \mathrm{Ct}$ method was used to examine the relative expression of VEGF in CTCs-enriched fraction compared to the CTCs-depleted fraction. Equal RNA amount from each fraction was processed for RT-PCR and Real-Tme PCR. Ct is the Real-Time PCR sample threshold cycle and $\Delta \mathrm{Ct}$ is $\left.\left(\mathrm{Ct}_{(\mathrm{CTCs})}\right)^{-} \mathrm{Ct}_{(\mathrm{PBMCs})}\right)$, PBMCs are the Peripheral Blood Mononuclear Cells (the CTCs-depleted fraction).

aggressive tumor biology as indicated by the significant correlation between VEGF expression, lymph node metastasis, distant metastasis and poorer survival.

In the present study, VEGF expression in CTCs was further confirmed by quantitative real-time RT-PCR in immunomagnetically separated CTCs. Indeed, CTCs from four out of 10 patients expressed both VEGF mRNA and VEGF protein. Immunofluorescence experiments further revealed VEGF protein expression in three additional patients who did not express VEGF mRNA. This discrepancy could be attributed to the different sensitivities of the used assays; alternatively, it is possible that this growth factor could be absorbed from the serum.

HIF-1 $\alpha$ induces VEGF expression in cells under hypoxic conditions by binding to VEGF promoter [8,33]. Recent studies have shown that HIF-1 $\alpha$ is increased in various carcinomas and its presence is associated with poor prognosis and resistance to therapy $[16,34,35]$. Focal adhesion kinase (FAK) is also important in promoting VEGF-induced tumor angiogenesis [13] whereas the expression of HIF-1 $\alpha$ depends on FAK and $\mathrm{Pl}-3$ kinase activation in cancer cells [17]. In order to characterize the possible functional pathways leading to the observed VEGF expression in CTCs we investigated the expression of HIF- $1 \alpha$ and pFAK in patients' CTCs. Double staining experiments revealed that HIF- $1 \alpha$ was expressed in $76 \%$ of breast cancer patients and this expression was observed both in the cytoplasm and the nucleus although, in general, cytospin preparations are not the optimal material to study the intracellular distribution of molecules. The intracellu- lar distribution reported in this study refers to what it was observed using confocal laser scanning microscopy (Figure 2B). Double (VEGF/HIF-1 $\alpha$ ) and triple (VEGF/HIF-1 $\alpha / \mathrm{CK}$ ) staining immunofluoresence experiments after immunomagnetic enrichment of CTCs, revealed that these molecules can be co-localized in the same cell (Figure 3BII and 3CII). These findings denote a possible interaction between VEGF and HIF- $1 \alpha$ in CTCs as it has already been shown in cancer cells [16]. In addition, our experiments revealed that FAK was phosphorylated in the CTCs of the majority (92\%) of the examined CK-positive metastatic breast cancer patients. These results are in agreement with our previously published data from early breast cancer patients [18]. Furthermore, all patients with pFAK-positive CTCs also had detectable HIF-1 $\alpha$ positive CTCs (Spearman's correlation analysis $P<0.001$ ), suggesting the presence of a functional pathway leading to VEGF production.

VEGFR2 is the principal receptor through which VEGFs exert their mitogenic and chemotactic effects $[8,36]$. In breast cancer cell lines VEGF regulates cell growth and survival via an autocrine loop including the phosphorylation of Akt and ERK1/ $2[15,37]$. We have recently shown that Akt was strongly activated in CTCs both in patients with early and metastatic disease [19]; however, there are no data in the literature concerning the expression of VEGFR2 in CTCs. The results of the current study demonstrate that VEGFR2 was expressed in CTCs of $47 \%$ of breast cancer patients. It is interesting to note that the proportion of CTCs expressing VEGF or VEGFR2 in all the tested patients was almost identical (73\% and $71 \%$, respectively; Figure $1 \mathrm{C}$ ); in addition, there was a statistically significant correlation between the number of CTCs expressing VEGF and VEGFR2 $(P=0.042)$. These data strongly suggest that patients' CTCs may both produce and consume VEGF through a putative autocrine mechanism. This assumption is further supported by the observed co-expression of these molecules (Figures $3 \mathrm{BI}$ and $3 \mathrm{Cl}$ ) in CTCs after immunomagnetic enrichment and double (VEGF/VEGFR2) or triple (VEGF/VEGFR/CK) staining experiments.

Our results also confirmed the heterogeneity of CTCs. Indeed, the molecules evaluated in the present study were not expressed in all examined CTCs, even not among those identified in the same patient. This may explain the fate and the metastatic potential of cells presenting such a phenotypic diversity.

\section{Conclusions}

The data reported in the present study demonstrate that CTCs from patients with metastatic breast cancer express VEGF both at mRNA and protein level. The production of VEGF is probably under the regulation of HIF- $1 \alpha$ and/or $\mathrm{pFAK}$ as suggested by the observed significant correlation between the expression of these molecules. Moreover, we have also demonstrated that VEGFR2 is also expressed on CTCs' mem- 
brane suggesting an autocrine loop involving VEGF and VEGFR2. The biologic significance of the presence of angiogenic molecules on CTCs is currently unknown. However, based on previous evidence, one might hypothesize that angiogenic pathways present on CTCs could result in evasion of apoptosis, enhancement of metastatic potential [10] and resistance to endocrine therapy [38]. In addition, the expression of angiogenic molecules on CTCs could be indicative of the tumor dependence on angiogenesis. Accordingly, antiVEGF and/or anti-VEGFR2 targeting agents might have therapeutic implications in patients with VEGF- and VEGFR2expressing CTCs.

\section{Competing interests}

The authors declare that they have no competing interests.

\section{Authors' contributions}

GK participated in the design and coordination of the study. She performed immunofluoresence experiments as well as the immunomagnetic separations and the cell cultures. She performed reverse transcription (RT) reactions. She also drafted the manuscript. HM performed RNA extraction. VG performed some immunofluoresence experiments. MP collected all the clinicopathological characteristics of the patients. She also performed some immunofluoresence experiments. AS performed quantitative real-time RT-PCR. EL participated in the coordination of the study and helped to draft the manuscript. VG provided general support and participated in study design. He also drafted the manuscript, DM helped to draft the manuscript and SA participated in the coordination of the study and help drafted the manuscript.

\section{Additional files}

The following Additional files are available online:

\section{Additional file 1}

A table listing the number of VEGF, VEGFR2 and HIF$1 \alpha$-positive CTCs in patients with breast cancer. It is named as Supplementary Table S1.

See http://www.biomedcentral.com/content/

supplementary/bcr2452-S1.doc

\section{Additional file 2}

A table listing the antibodies and the corresponding dilutions utilized in the present study. It is named as Supplementary Table S2.

See http://www.biomedcentral.com/content/ supplementary/bcr2452-S2.doc

\section{Additional file 3}

A diagram presenting the quantification of VEGF, VEGFR2, HIF- $1 \alpha$ and pFAK expression in breast cancer cell lines. It is named as Supplementary diagram. See http://www.biomedcentral.com/content/ supplementary/bcr2452-S3.doc

\section{Acknowledgements}

This work was supported by Research Grants from Cretan Association of Biomedical Research (CABR).

\section{References}

1. Braun S, Harbeck N: Molecular markers of metastasis in breast cancer: current understanding and prospects for novel diagnosis and prevention. Expert Rev Mol Med 2001, 3:1-14.

2. Pantel K, Muller V, Auer M, Nusser N, Harbeck N, Braun S: Detection and clinical implications of early systemic tumor cell dissemination in breast cancer. Clin Cancer Res 2003, 9:6326-6334.

3. Pantel K, Cote RJ, Fodstad O: Detection and clinical importance of micrometastatic disease. J Natl Cancer Inst 1999, 91:1113-1124.

4. Stathopoulou A, Vlachonikolis I, Mavroudis D, Perraki M, Kouroussis C, Apostolaki S, Malamos N, Kakolyris S, Kotsakis A, Xenidis N, Reppa D, Georgoulias V: Molecular detection of cytokeratin-19positive cells in the peripheral blood of patients with operable breast cancer: evaluation of their prognostic significance. $J$ Clin Oncol 2002, 20:3404-3412.

5. Xenidis N, Perraki M, Kafousi M, Apostolaki S, Bolonaki I, Stathopoulou A, Kalbakis K, Androulakis N, Kouroussis C, Pallis T, Christophylakis C, Argyraki K, Lianidou ES, Stathopoulos S, Georgoulias V, Mavroudis D: Predictive and prognostic value of peripheral blood cytokeratin-19 mRNA-positive cells detected by real-time polymerase chain reaction in node-negative breast cancer patients. J Clin Oncol 2006, 24:3756-3762.

6. Bidard FC, Vincent-Salomon A, Sigal-Zafrani B, Dieras V, Mathiot C, Mignot L, Thiery JP, Sastre-Garau X, Pierga JY: Prognosis of women with stage IV breast cancer depends on detection of circulating tumor cells rather than disseminated tumor cells. Ann Oncol 2008, 19:496-500.

7. Leung DW, Cachianes G, Kuang WJ, Goeddel DV, Ferrara N Vascular endothelial growth factor is a secreted angiogenic mitogen. Science 1989, 246:1306-1309.

8. Pradeep CR, Sunila ES, Kuttan G: Expression of vascular endothelial growth factor (VEGF) and VEGF receptors in tumor angiogenesis and malignancies. Integr Cancer Ther 2005, 4:315-321.

9. Liang Y, Brekken RA, Hyder SM: Vascular endothelial growth factor induces proliferation of breast cancer cells and inhibits the anti-proliferative activity of anti-hormones. Endocr Relat Cancer 2006, 13:905-919.

10. Bachelder RE, Wendt MA, Mercurio AM: Vascular endothelial growth factor promotes breast carcinoma invasion in an autocrine manner by regulating the chemokine receptor CXCR4. Cancer Res 2002, 62:7203-7206.

11. Zhang $X, X u$ WH, Ge YL, Hou L, Li Q: Effect of siRNA transfection targeting VEGF gene on proliferation and apoptosis of human breast cancer cells. Xi Bao Yu Fen Zi Mian Yi Xue Za Zhi 2007, 23:14-17.

12. Mohammed RA, Green A, El-Shikh S, Paish EC, Ellis IO, Martin SG: Prognostic significance of vascular endothelial cell growth factors $-A,-C$ and $-D$ in breast cancer and their relationship with angio- and lymphangiogenesis. Br J Cancer 2007, 96:1092-1100.

13. Mitra SK, Schlaepfer DD: Integrin-regulated FAK-Src signaling in normal and cancer cells. Curr Opin Cell Biol 2006, 18:516-523.

14. Mitra SK, Mikolon D, Molina JE, Hsia DA, Hanson DA, Chi A, Lim ST, Bernard-Trifilo JA, llic D, Stupack DG, Cheresh DA, Schlaepfer 
DD: Intrinsic FAK activity and Y925 phosphorylation facilitate an angiogenic switch in tumors. Oncogene 2006, 25:5969-5984

15. Laramee M, Chabot $C$, Cloutier M, Stenne R, Holgado-Madruga M, Wong AJ, Royal I: The scaffolding adapter Gab1 mediates vascular endothelial growth factor signaling and is required for endothelial cell migration and capillary formation. $\mathrm{J} \mathrm{Bio/} \mathrm{Chem}$ 2007, 282:7758-7769.

16. Harris AL: Hypoxia--a key regulatory factor in tumour growth. Nat Rev Cancer 2002, 2:38-47.

17. Liu L, Ning X, Sun L, Shi Y, Han S, Guo C, Chen Y, Sun S, Yin F, Wu K, Fan D: Involvement of MGr1-Ag/37LRP in the vincristine-induced HIF-1 expression in gastric cancer cells. Mol Cell Biochem 2007, 303:151-160.

18. Kallergi G, Mavroudis D, Georgoulias V, Stournaras C: Phosphorylation of $\mathrm{FAK}, \mathrm{PI}-3 \mathrm{~K}$, and impaired actin organization in CKpositive micrometastatic breast cancer cells. Mol Med 2007, 13:79-88.

19. Kallergi G, Agelaki S, Kalykaki A, Stournaras C, Mavroudis D, Georgoulias V: Phosphorylated EGFR and PI-3K/Akt signaling kinases are expressed in circulating tumor cells of breast cancer patients. Breast Cancer Res 2008, 10:R80.

20. Takata K, Morishige K, Takahashi T, Hashimoto K, Tsutsumi S, Yin L, Ohta T, Kawagoe J, Takahashi K, Kurachi H: Fasudil-induced hypoxia-inducible factor-1\{alpha\} degradation disrupts a hypoxia-driven vascular endothelial growth factor autocrine mechanism in endothelial cells. Mol Cancer Ther 2008, 7:1551-1561.

21. Stathopoulou A, Gizi A, Perraki M, Apostolaki S, Malamos N, Mavroudis D, Georgoulias V, Lianidou ES: Real-time quantification of CK-19 mRNA-positive cells in peripheral blood of breast cancer patients using the lightcycler system. Clin Cancer Res 2003, 9:5145-5151.

22. Meng S, Tripathy D, Frenkel EP, Shete $S$, Naftalis EZ, Huth JF, Beitsch PD, Leitch M, Hoover S, Euhus D, Haley B, Morrison L, Fleming TP, Herlyn D, Terstappen LW, Fehm T, Tucker TF, Lane N, Wang J, Uhr JW: Circulating tumor cells in patients with breast cancer dormancy. Clin Cancer Res 2004, 10:8152-8162.

23. Naume B, Borgen E, Nesland JM, Beiske K, Gilen E, Renolen A, Ravnas G, Qvist H, Karesen R, Kvalheim G: Increased sensitivity for detection of micrometastases in bone-marrow/peripheralblood stem-cell products from breast-cancer patients by negative immunomagnetic separation. Int J Cancer 1998, 78:556-560.

24. Zygalaki E, Stathopoulou A, Kroupis C, Kaklamanis L, Kyriakides Z, Kremastinos D, Lianidou ES: Real-time reverse transcriptionPCR quantification of vascular endothelial growth factor splice variants. Clin Chem 2005, 51:1518-1520.

25. Tai SK, Tan OJ, Chow VT, Jin R, Jones JL, Tan PH, Jayasurya A, Bay $\mathrm{BH}$ : Differential expression of metallothionein 1 and 2 isoforms in breast cancer lines with different invasive potential: identification of a novel nonsilent metallothionein-1H mutant variant. Am J Pathol 2003, 163:2009-2019.

26. Kagara N, Tanaka N, Noguchi S, Hirano T: Zinc and its transporter ZIP10 are involved in invasive behavior of breast cancer cells. Cancer Sci 2007, 98:692-697.

27. Kattan Z, Minig V, Leroy P, Dauca M, Becuwe P: Role of manganese superoxide dismutase on growth and invasive properties of human estrogen-independent breast cancer cells. Breast Cancer Res Treat 2008, 108:203-215.

28. Zagzag D, Nomura M, Friedlander DR, Blanco CY, Gagner JP, Nomura N, Newcomb EW: Geldanamycin inhibits migration of glioma cells in vitro: a potential role for hypoxia-inducible factor (HIF-1alpha) in glioma cell invasion. J Cell Physiol 2003, 196:394-402.

29. Livak KJ, Schmittgen TD: Analysis of relative gene expression data using real-time quantitative PCR and the 2(-Delta Delta C(T)) Method. Methods 2001, 25:402-408.

30. Lobodasch K, Frohlich F, Rengsberger M, Schubert R, Dengler R, Pachmann U, Pachmann K: Quantification of circulating tumour cells for the monitoring of adjuvant therapy in breast cancer: an increase in cell number at completion of therapy is a predictor of early relapse. Breast 2007, 16:211-218.

31. Klein CA, Blankenstein TJ, Schmidt-Kittler O, Petronio M, Polzer B, Stoecklein NH, Riethmuller G: Genetic heterogeneity of single disseminated tumour cells in minimal residual cancer. Lancet 2002, 360:683-689.
32. Paterlini-Brechot $P$, Benali NL: Circulating tumor cells (CTC) detection: Clinical impact and future directions. Cancer Lett 2007, 253:180-204.

33. Levy AP, Levy NS, Wegner S, Goldberg MA: Transcriptional regulation of the rat vascular endothelial growth factor gene by hypoxia. J Biol Chem 1995, 270:13333-13340.

34. Zhong $\mathrm{H}$, De Marzo AM, Laughner E, Lim M, Hilton DA, Zagzag D, Buechler P, Isaacs WB, Semenza GL, Simons JW: Overexpression of hypoxia-inducible factor 1alpha in common human cancers and their metastases. Cancer Res 1999, 59:5830-5835.

35. Talks KL, Turley H, Gatter KC, Maxwell PH, Pugh CW, Ratcliffe PJ, Harris AL: The expression and distribution of the hypoxiainducible factors HIF-1alpha and HIF-2alpha in normal human tissues, cancers, and tumor-associated macrophages. $A m ~ J$ Pathol 2000, 157:411-421.

36. Neufeld G, Tessler S, Gitay-Goren H, Cohen T, Levi BZ: Vascular endothelial growth factor and its receptors. Prog Growth Factor Res 1994, 5:89-97.

37. Weigand M, Hantel P, Kreienberg R, Waltenberger J: Autocrine vascular endothelial growth factor signalling in breast cancer Evidence from cell lines and primary breast cancer cultures in vitro. Angiogenesis 2005, 8:197-204.

38. Aesoy R, Sanchez BC, Norum JH, Lewensohn R, Viktorsson $K$ Linderholm B: An autocrine VEGF/VEGFR2 and p38 signaling loop confers resistance to 4-hydroxytamoxifen in MCF-7 breast cancer cells. Mol Cancer Res 2008, 6:1630-1638. 